\title{
A laparoscopic duodenojejunostomy for a duodenal obstruction from lymphoma
}

\author{
J. Cyriac, L. Klein
}

University of Toronto, Minimally Invasive Surgery Program

Received: 31 December 2005/Accepted: 12 March 2006/Online publication: 28 December 2006

\begin{abstract}
A patient with lymphoma in one-third of the duodenum causing a duodenal obstruction is described. The patient had a partial response with chemotherapy, but still was obstructed and unable to eat. He was losing weight, and chemotherapy had to be stopped. A gastrostomy tube was inserted for drainage because the stomach was quite distended. A jejunostomy tube was passed through the gastrostomy tube for feeding, but the patient did not tolerate the feeding. A laparoscopic bypass of the duodenumduodenal obstruction (from duo-
\end{abstract}

denum to jejunum) for this patient is shown on a video. The patient did very well after this bypass was provided. He was able to tolerate an oral diet on postoperative day 2 , and on postoperative day 4 , he was discharged home. He has since resumed chemotherapy, and is doing well, at this writing, 2 months after surgery.

Key words: Surgical - Technical, Abdominal, Bowel, General - Cancer, Cancer, General - Endoscopy 